\title{
Médiévales
}

Langues, Textes, Histoire

76 | printemps 2019

Du nouveau en archives

\section{L'innovation documentaire à la fin du Moyen Âge}

\section{Harmony Dewez}

\section{(2) OpenEdition}

Journals

Édition électronique

URL : https://journals.openedition.org/medievales/9750

DOI : 10.4000/medievales. 9750

ISSN : 1777-5892

\section{Éditeur}

Presses universitaires de Vincennes

\section{Édition imprimée}

Date de publication : 5 septembre 2019

Pagination : 5-10

ISBN : 978-2-37924-028-7

ISSN : 0751-2708

\section{Référence électronique}

Harmony Dewez, «L'innovation documentaire à la fin du Moyen Âge », Médiévales [En ligne], 76 | printemps 2019, mis en ligne le 01 janvier 2021, consulté le 22 avril 2022. URL : http://

journals.openedition.org/medievales/9750; DOI : https://doi.org/10.4000/medievales.9750 
Harmony Dewez

\section{L'innovation documentaire à la fin du Moyen Âge}

Aujourd'hui bien intégrée dans la démarche historienne, l'étude des pratiques de l'écrit est une façon d'envisager l'artefact « document » à la fois comme objet d'histoire et comme facteur d'interprétation du texte ${ }^{1}$. Ceci permet de renouveler notre compréhension des mécanismes sociaux à travers l'analyse de la vie du manuscrit et des initiatives individuelles qui l'ont produit et transformé ; à travers une réflexion sur les formes d'écrits et leur relation aux activités des hommes ; à travers la prise en compte du rôle de l'écrit dans les communautés et des conséquences sur les groupes humains d'une familiarité transformée avec ce médium. Ces trois aspects figuraient d'emblée dans l'ouvrage séminal de Michael Clanchy, que l'on ne pourra jamais trop citer tant son propos est pertinent ${ }^{2}$. Son travail n'a toutefois pas fait école dans l'immédiat : le courant de la «scripturalité » et des «pratiques de l'écrit » a eu plus de succès sur le continent qu'en GrandeBretagne, et ce n'est qu'à partir des années 1990 que cette approche s'est véritablement diffusée ${ }^{3}$. Le dossier sur les Pratiques de l'écrit paru en 2008

1. Voir par exemple L. KuCHENBUCH, U. KLEINEéd., Textus im Mittelalter. Komponenten und Situationen des Wortgebrauchs im schriftsemantischen Feld, Göttingen, 2006. Le présent dossier est issu d'une journée d'études qui s'est déroulée à Namur le 19 novembre 2018, intitulée «Formes et mécanismes de l'innovation documentaire dans les écrits pragmatiques en Europe du Nord-Ouest (XIII $-\mathrm{XV}^{\mathrm{e}}$ siècles) ». Elle fut organisée dans le cadre d'un postdoctorat Move-In Louvain à l'Université de Namur, qu'il convient de remercier, ainsi que le FRS/FNRS. Je remercie très chaleureusement toute l'équipe du PraME : Jean-François Nieus, Xavier Hermand, Étienne Renard et Nicolas Ruffini pour leurs conseils et leur soutien ; Émilie Debu, Manon Vilain pour leur aide logistique. Je remercie également Paul Bertrand qui a généreusement accepté d'en faire les conclusions.

2. M. ClANCHY, From Memory to Written Record, England 1066-1307, Londres, 1979, Chichester, 2013 [3 $3^{\mathrm{e}}$ éd.].

3. Citons la thèse de P. CHASTANG, Lire, écrire, transcrire. Le travail des rédacteurs de cartulaires en Bas-Languedoc (XI'-XIII ${ }^{e}$ siècles), Paris, 2001 ; ou encore l'article de 
dans cette même revue, coordonné par Pierre Chastang et Étienne Anheim, témoignait de la place nouvelle de celles-ci dans l'outillage du médiéviste, patiemment acquise grâce à cette génération d'historiens qui ont contribué par leur enseignement et leurs travaux à faire connaître l'importance du médium comme facteur d'interprétation des textes historiques, et comme fait lui-même ${ }^{4}$. Le large XIII ${ }^{\mathrm{e}}$ siècle qui va de 1180 à 1330 est désormais considéré comme la période charnière de ce que l'on a coutume d'appeler la « révolution documentaire », la « révolution de l'écrit », ou le «tournant pragmatique », phase d'essor majeur de la production scripturale, tant d'un point de vue quantitatif que par les types d'activités et d'informations qu'elle concerne, au point de marquer le changement de paradigme qualifié si justement par Michael Clanchy de passage de la mémoire au document écrit, c'est-à-dire d'une société de l'oralité, dans laquelle les structures sociales reposaient sur les relations d'homme à homme, à une société dont les structures sociales sont désormais en partie médiatisées par le biais de l'écrit. Dix ans après, la nécessité de prendre en compte le support matériel des documents, la structure visuelle et logique du texte, les fonctions possibles des modes de communication et le statut de l'écrit comme vecteur d'information n'est plus à démontrer et se pratique de façon plus évidente, notamment par ceux qui y ont été familiarisés dès leur formation. Certains jalons ont été posés ${ }^{5}$, mais, malgré le temps écoulé et les résultats acquis, les questions abondent toujours nombreuses. La plus pressante est peut-être celle-ci : quel est le devenir de la « révolution documentaire »? Après le « tournant » des années 1180-1330, temps de changement de paradigme marqué par l'application de l'écrit à de nouveaux domaines d'activité, transformant radicalement les modes de justification de l'information au

J. Morsel, «Ce qu'écrire veut dire au Moyen Âge... Observations préliminaires à une étude de la scripturalité médiévale », Memini. Travaux et documents de la Société des études médiévales du Québec, 4 (2000), p. 3-43.

4. Médiévales, 56 (2009). Ont contribué à ce dossier Étienne Anheim, Pierre Chastang, Alice Rio, François Bougard, Laurent Morelle, Paul Bertrand et Roger Chartier.

5. Pour un bilan historiographique, voir S. BArRet, D. Stutzmann, G. Vogeler, «Introduction », dans S. BARRET, D. STUTZMANN, G. VogelER éd., Ruling the Script in the Middle Ages. Formal Aspects of Written Communication (Books, Charters, and Inscriptions), Turnhout, 2016, p. 1-24 ; H. DEWEZ, «Introduction », dans H. DEWEZ, L. TRYOEN éd., Administrer par l'écrit au Moyen Âge (XII -XVe siècle), Paris, 2019, p. 9-20. Pour d'autres exemples de travaux récents, voir P. CHASTANG, La Ville, le gouvernement et l'écrit à Montpellier, Paris, 2013 ; O. MATTÉONI, P. BECK, éd., Classer, dire, compter. Discipline du chiffre et fabrique d'une norme comptable à la fin du Moyen Âge, Paris, 2015 ; B. GRÉVIN, A. MAIREY éd., Le Moyen Âge dans le texte. Cinq ans d'histoire textuelle au Laboratoire de médiévistique occidentale de Paris, Paris, 2016 ; M. MOSTERT éd., Organizing the Written Word. Scripts, Manuscripts and Texts, Turnhout, 2017. Il faut saluer l'ouvrage de P. BERTRAND, Les Écritures ordinaires. Sociologie d'un temps de révolution documentaire (1250-1350), Paris, 2015, le seul à entreprendre une synthèse des résultats. 
sein des sociétés médiévales, qu'advient-il des mécanismes de diffusion des pratiques de l'écrit et de leur impact sur le tissu social et les institutions ?

Les mutations du «tournant pragmatique » reposent en grande partie sur la création de nouveaux documents. Ce processus tient une place complexe dans les dynamiques sociales et institutionnelles : les producteurs d'écrits, confrontés à leur production et aux nouveaux usages qu'elle permet ou non, peuvent engager des actualisations du support qui renforcent une pratique ou, au contraire, l'abandonner. La production documentaire servant de révélateur aux pratiques dont elle force l'expression dans le cadre contraint de l'écrit influence en retour ceux qui l'initient. C'est ainsi que la mise par écrit et la mise en écrit des documents - la Verschriftlichung de l'école de Münster -, en association avec le développement de pratiques d'archivage plus ou moins systématiques selon les contextes, catalysent l'émergence de nouvelles catégories de pensée, dont la pensée comptable est un exemple. Ces phénomènes président à la constitution de mémoires documentaires - mémoires judiciaires, par exemple, qui transforment la pratique du droit, comme dans le cas de la Common Law anglaise, reposant sur le développement des Year Books, des sélections d'affaires juridiques traitées par les cours royales copiées et archivées annuellement de façon continue entre 1268 et 1535 pour servir à l'élaboration du précédent ${ }^{6}$. De nouveaux types documentaires se diffusent également par imitation : la pratique de sélectionner des cas se retrouve dans la justice seigneuriale, dont les sujets requièrent toujours plus de recherches de références anciennes pouvant justifier leurs procès.

Dans les derniers siècles du Moyen Âge, l'essor quantitatif de la production et de l'archivage des écrits de la pratique transforme les modalités d'évolution du paysage documentaire. Cet essor quantitatif n'est pas un processus mécanique, mais résulte de choix qualitatifs qui ne sont pas nécessairement les mêmes que ceux des époques précédentes, puisqu'ils s'inscrivent dans un contexte où la gestion de l'information se fait selon des termes qui préparent le terreau du changement de paradigme suivant, celui de l'imprimerie et de la reproductibilité des supports, y compris pragmatiques. Dans ce contexte d'une société dans laquelle la communication écrite a pris une place croissante en tant que rouage des interactions sociales, de nouveaux besoins et de nouveaux usages ont émergé, nourris par la formation des États, le développement des universités, des choix techniques comme l'utilisation du papier et, de façon générale, l'instauration d'une «mentalité de l'écrit » signifiant que, même si l'on

6. Voir A. Musson, Medieval Law in Context. The Growth of Legal Consciousness from Magna Carta to the Peasants'Revolt, Manchester, 2001 ; A. Musson, W. M. ORMROD, The Evolution of English Justice. Law, Politics and Society in the Fourteenth Century, Basingstoke, 1999. 
ne savait pas lire ou écrire, on possédait une familiarité avec le document écrit, parce que l'on y était confronté quotidiennement ${ }^{7}$. Les processus de mise par écrit d'actions auparavant confinées à l'oral continuent de se produire et ainsi d'entraîner l'apparition de nouveaux types documentaires, tandis que les quantités d'informations déjà consignées par écrit, parfois dans des types documentaires parfaitement établis, subissent elles aussi des influences transformatives, que ce soit dans leur organisation graphique, dans leur mode de rédaction ou dans celui de leur sélection. L'étude des pratiques de l'écrit à la fin du Moyen Âge doit donc s'attacher à identifier ces changements d'expression documentaire, à exposer les façons nouvelles de traiter l'information et à expliciter les réalités sociales qui les sous-tendent, au-delà de certains semblants de permanence.

L'apparente stabilité d'un genre documentaire ancien tel que celui du censier, du cartulaire, voire d'un genre plus récent comme celui du compte, masque en effet des évolutions où l'on retrouve la double problématique des changements à l'échelle du document et des changements à l'échelle de leur usage par les groupes sociaux. Ainsi, si le cartulaire en tant qu'ensemble de copies de chartes perdure à l'époque moderne, de nouvelles formes de « cartularisation» témoignent de l'essor de nouveaux moyens de légitimer les droits et la possession. En Angleterre, on compose à partir du XIII ${ }^{\mathrm{e}}$ siècle des ensembles de copies de documents qui, pour l'essentiel, ne sont pas des chartes, mais des actes issus de diverses institutions - chancelleries, cours de justice urbaines ou royales, administrations locales. Ces ouvrages, qui suivent la logique du cartulaire en l'appliquant à d'autres objets, commencent à être identifiés dans la seconde moitié du XIV ${ }^{\mathrm{e}}$ siècle comme une nouvelle catégorie documentaire, celle du liber evidenciarum - book of evidences, ou livre de « preuves »- qui répond au besoin de créer des outils pour gérer ces nouveaux types documentaires, souvent d'origine royale, permettant la défense des droits seigneuriaux - statuts juridiques, feet of fines, writs, quittances, extraits des rôles de l'Échiquier et autres ${ }^{8}$.

Les transformations de l'écrit aux $\mathrm{XIV}^{\mathrm{e}}$ et $\mathrm{XV}^{\mathrm{e}}$ siècles apportent donc de nouvelles façons de faire, de nouveaux objets documentaires, qui matérialisent et influencent de nouvelles façons d'agir, pour reprendre la

7. M. ClanCHY, From Memory to Written Record...

8. Voir par exemple le Livre de John Blanchard, archidiacre de Worcester à la fin du $\mathrm{XIV}^{\mathrm{e}}$ siècle, étudié par Antoine Thiry : A. THIRY, Étude d'un cartulaire anglais de la fin du XIVe siècle : le registre de maître John Blanchard, archidiacre de Worcester, sur les droits de son manoir à Barford St Martin, mémoire de master inédit sous la direction de P. BERTRAND, Université Catholique de Louvain-la-Neuve, 2018. Notons que ceci incite à repenser ce que l'on désigne par «ses propres documents » dans la définition du cartulaire par la Commission de diplomatique en 1984, définition selon laquelle il s'agit d'un « recueil de copies de ses propres documents ». 
théorie de l'action développée par Pierre Chastang et Étienne Anheim9. Saisir les transformations sociales du bas Moyen Âge ne peut se faire sans envisager les mutations du paysage documentaire et c'est ce que nous proposons d'aborder dans ce dossier à partir d'un ensemble d'études de cas issues de contextes croisés, touchant aux individus comme aux institutions, au monde des clercs comme à celui des laïcs. Pour qualifier ces transformations, nous avons choisi le terme d'innovation. Non au sens que certains seraient tentés de lui donner d'une inéluctable marche vers une rationalité comprise comme moderne, mais parce qu'il permet de suggérer les processus de renouvellement documentaire une fois le paradigme de l'écrit à usage administratif, économique et judiciaire entré dans les mœurs. Celle-ci s'exprime, ici encore, par l'émergence combinée de plusieurs facteurs, à plusieurs échelles : nouveaux gestes, nouvelles pratiques, nouveaux acteurs de l'écrit, qui aboutissent à la genèse de formes originales dont les mécanismes de création et, le cas échéant, de diffusion dépendent des contextes dans lesquels ils apparaissent. L'espace considéré va également en ce sens : traversé de circulations, d'échanges, de réseaux, durement marqué par les conflits qui rythment les derniers siècles du Moyen Âge, lesquels imposent leurs interactions contraintes qui rassemblent autant qu'elles divisent. Les articles de ce dossier aborderont plus particulièrement le Nord de la France, le Pays de Galles et l'espace normand, à travers le prisme de différents types de producteurs d'écrits, qu'il s'agisse du monde des villes, des monastères, des princes, ou des individus.

Ces travaux mettent en évidence plusieurs thèmes centraux de l'innovation documentaire à la fin du Moyen Âge. De nouveaux modes d'élaboration de l'écrit se normalisent : l'essor des pratiques d'enregistrement en est l'une des principales facettes. La construction de nouveaux outils se fait aussi par expérimentation, voire «bricolage » pour reprendre l'expression d'Emmanuel Melin, reflétant le caractère progressif et cumulatif de l'innovation documentaire à partir d'essais, de « brouillons ». Les nouvelles formes sont souvent issues de la combinaison de types documentaires plus anciens, refondus en des collections administratives répondant mieux aux besoins. Observer la création et la consolidation de nouveaux types documentaires, comme dans le cas des livres et registres urbains du Nord de la France, invite à penser le caractère conjoint des dynamiques d'institutionnalisation administrative et de maîtrise des flux informationnels. Prolongement visuel, matériel, pérenne ou semi-pérenne de la pensée et de la voix, le document écrit transforme la dimension de l'action humaine, et particulièrement de l'action collective dont il permet une autre forme de coordination dans l'espace et le temps. La dialectique entre

9. É. AnHeim, P. Chastang, « Les pratiques de l'écrit dans les sociétés médiévales (VI ${ }^{\mathrm{e}}-\mathrm{XIII}{ }^{\mathrm{e}}$ siècle) », Médiévales, 56 (2009), p. 5-10. 
initiative individuelle et contrainte institutionnelle, politique ou logistique confirme un équilibre variable dans la part des unes et des autres. Dans le cas des écritures de gestion patrimoniale individuelle ou familiale, c'est le constat de logiques partagées par des acteurs sans relation, comme l'illustre la centralité du noyau « censier » dans la composition de nouveaux livres administratifs. L'imposition ou la diffusion de modèles, que cela touche les municipalités ou les chancelleries princières, sert des objectifs politiques ; mais il ne faut pas oublier la part du savoir individuel de chaque scribe et la possible organisation collective du travail de production documentaire. La tension entre initiative individuelle et mise en œuvre collective est d'autant plus complexe que de nouveaux acteurs de l'écrit entrent en jeu et que la production documentaire s'accroît et se systématise.

Malgré le caractère inégal, non linéaire et incomplet de ce phénomène, il n'en reste pas moins que l'on peut voir, $\mathrm{du} X \mathrm{XIII}{ }^{\mathrm{e}}$ au XV $\mathrm{XV}^{\mathrm{e}}$ siècle, des paysages documentaires se transformer à plusieurs échelles, qu'il s'agisse de la mise en page, de la typologie, de l'organisation de la production ou de leur usage. Ces transformations elles-mêmes, mais peut-être plus encore leurs mécanismes et les modalités de leur mise en œuvre, servent de révélateur aux mutations sociales qu'elles accompagnent, l'une nourrissant l'autre et réciproquement.

Harmony Dewez - Université de Poitiers/CESCM 